\title{
SILICON-ON-SILICON (SOS): A NEW CMOS COMPATIBLE LOW-TEMPERATURE MEMS PROCESS USING PLASMA ACTIVATED FUSION BONDING
}

\author{
Tzeno Galchev, Warren C. Welch III, and Khalil Najafi \\ Center for Wireless Integrated Microsystems (WIMS) \\ University of Michigan, Ann Arbor, Michigan USA, 48109-2122
}

\begin{abstract}
This paper explores the use of dielectric barrier discharge (DBD) surface activated low-temperature wafer bonding in MEMS device fabrication. A new high aspect-ratio MEMS technology based on bonding two silicon wafers with an intermediate silicon dioxide layer at $400^{\circ} \mathrm{C}$ is presented. This Silicon-On-Silicon (SOS) process requires three masks and provides several advantages compared with Silicon-on-Glass (SOG) and Silicon-on-Insulator (SOI) processes, including better dimensional and etch profile control of narrow and slender MEMS structures. This is demonstrated by fabricating a $5 \mu \mathrm{m}$ wide $30 \mathrm{~mm}$ long beam. Additionally, by patterning the intermediate $\mathrm{SiO}_{2}$ insulation layer before bonding, footing is reduced without any extra processing, as compared to both SOG and SOI. All SOS process steps are CMOS compatible.
\end{abstract}

\section{INTRODUCTION}

Deep Reactive Ion Etch (DRIE) is a proven technology for creating high aspect-ratio structures. Silicon-On-Glass (SOG) [1] and Silicon-On-Insulator (SOI) are widely used processes which have been developed around DRIE [1,2]. In the case of SOG, a glass substrate is recessed, a silicon device wafer is anodicaly bonded to it, and MEMS devices are formed using DRIE from this silicon wafer. However, glass causes problems during DRIE, namely "footing" or "notching," and overheating. Although footing can be dealt with using shield metals [1], at the expense of process complexity, there is no effective way of removing the heat created during the etch because glass is a poor thermal conductor. This problem is compounded by the fact that a backing wafer must always be used because the glass is not strong enough to survive the pressurized helium cooling in a typical ICP chamber. In the case of SOI, already processed substrates, consisting of a silicon film separated from a silicon wafer using an intermediate buried oxide layer, are etched using DRIE and then released using an isotropic etch of the buried oxide layer. Although overheating is no longer a problem, the oxide creates the same footing problem as glass. Additional processing can be used to solve this by etching first from the back and removing the oxide layer underneath the devices before DRIE [3]. Alternatively, footing can be reduced by limiting the layout to having uniform width and varying the DRIE process parameters [4].

This paper presents a new process for high aspect-ratio MEMS devices based on low-temperature plasma activated fusion bonding. An oxidized silicon wafer is patterned to form silicon dioxide islands, and a second device wafer is bonded to it. DRIE is used to form and release the mechanical structures. Silicon-OnSilicon (SOS) processing has several advantages, when compared to SOG and SOI. Because the oxide is patterned before DRIE, footing problems can be minimized without additional fabrication steps, and the need for wet etching of the oxide after DRIE is removed; so the MEMS devices will not see any wet processing after release. Additionally, when the carrier wafer is silicon, not only is a backing wafer not needed, but also heating during etching is reduced. Third, the carrier wafer can undergo standard micro-

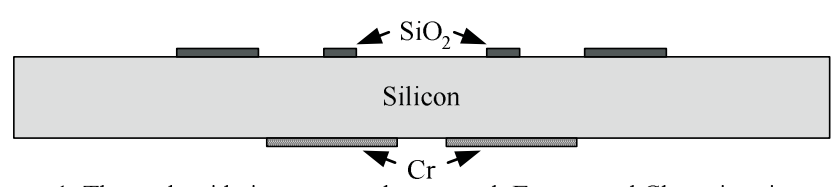

1. Thermal oxide is grown and patterned; Evaporated Chromium is deposited on the back for alignment.

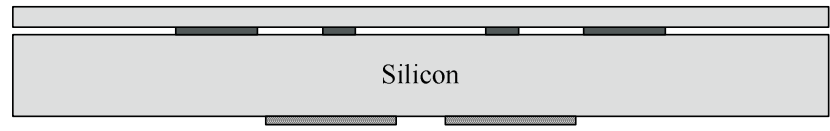

2. After cleaning and surface pretreatment of both wafers, they are bonded and annealed.

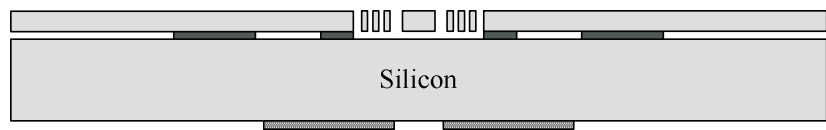

3. Devices are released using the Deep Reactive Ion Etch (DRIE) process.

Figure 1. SOS process flow

fabrication technologies before bonding. Fourth, the thermal mismatch between silicon and glass in the SOG process, which can add a temperature component to precision sensors, is eliminated in the SOS process where the carrier wafer is silicon. Fifth, the lowtemperature process allows the formation of buried feedthroughs using a variety of materials, including metals. Finally, SOS does offer the opportunity of achieving full integration because all process steps are CMOS compatible.

\section{FABRICATION AND RESULTS}

In its simplest form the SOS process consists of three masking steps. The process flow is shown in Figure 1. First, a $1 \mu \mathrm{m}$ wet thermal oxide is grown and patterned using RIE. The oxide serves as electrical insulation and bond sites for the device wafer. Alignment patterns made from $250 \AA$ of evaporated chromium are patterned on the back using lift-off. A thin $100 \mu \mathrm{m}$ electrically conductive $\mathrm{p}^{++}$silicon wafer is used for eventual creation of the MEMS devices. This wafer is dipped in BHF and rinsed in DI water. Both wafers are then pretreated using dielectric barrier discharge (DBD) atmospheric pressure $\mathrm{N}_{2}$ plasma in a SUSS MicroTec nanoPREP 200 system. The DBD method is described in Figure 2. The plasma is created with a $400 \mathrm{~W}$ AC bias and scanned across the wafers at a gap of $300 \mu \mathrm{m}$, while $50 \mathrm{slpm} \mathrm{N}_{2}$ flows between the electrodes. Plasma treatment creates molecular level surface modifications which increase the surface tension and make silicon hydrophilic [5]. The carrier wafer is megasonically cleaned and dried in a SUSSS MicroTec CL200 cleaner, while the more fragile device wafer, which is only $100 \mu \mathrm{m}$ thick, is hydrated in DI water for 5 minutes and blown dry with nitrogen. The two wafers are bonded in a SUSS MicroTec SB6e substrate bonder and annealed at $400^{\circ} \mathrm{C}$ for $4 \mathrm{hrs}$ while applying a pressure of 300 Torr. The devices are released using DRIE in a Surface Technology Systems ICP-RIE chamber.

A SEM of a fabricated die, consisting of two comb-drive resonators and two Pirani gauges, is shown in Figure 3. The same 
Electrode Assembly is

Scanned Across the Wafer

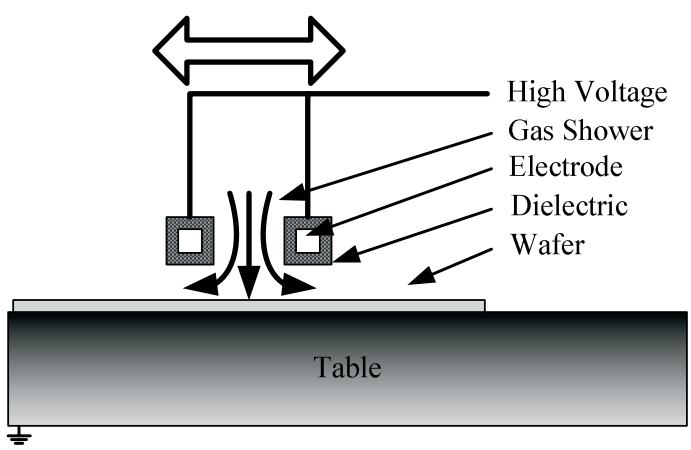

Figure 2. Dielectric Barrier Discharge creates a uniform plasma discharge due to a high frequency AC voltage applied between two electrodes. The discharge is scanned across the wafer surface. This process does not affect sensitive substrates.

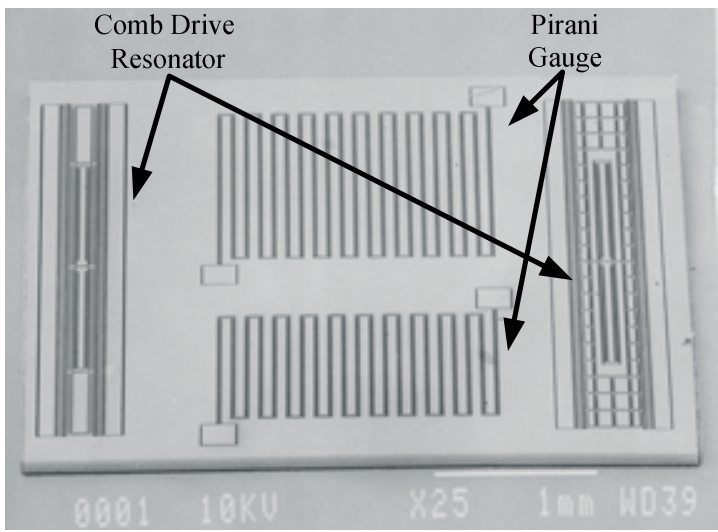

Figure 3. SEM of the fabricated SOS die. It consists of two comb dive resonators and two Pirani gauges.

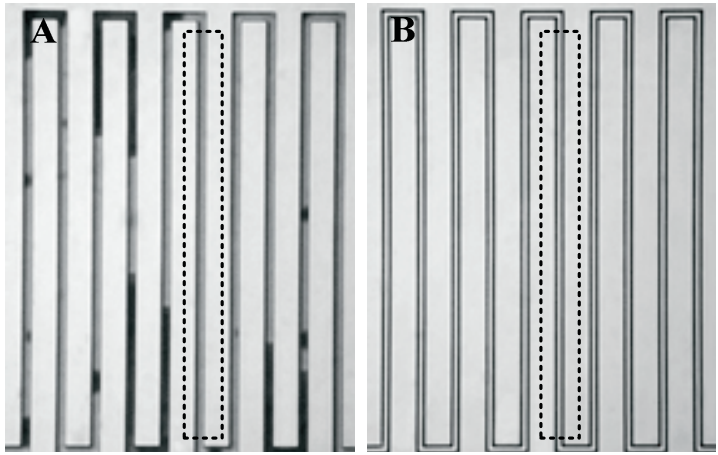

Figure 4. Close up photograph of one of the Pirani gauges made using SOG (a) and SOS (b). Devices made with SOS survive whereas the devices made with SOG are over etched due to the trapped heat during DRIE. The dark spots in A constitute areas where the underlying shield metal has been burned due to the intense heat.

mask set was used to make the same devices with the SOG process. The glass was recessed by $8 \mu \mathrm{m}$ before bonding. Additionally, both processes used the same DRIE recipe. The glass substrate was mounted on a silicon wafer for structural support during DRIE with thermally conductive grease. A close-up photo of one of the Pirani gauges is shown in Figure 4a SOG and Figure $4 \mathrm{~b}$ SOS. The Pirani gauge, which consists of $30 \mathrm{~mm}$ long $10 \mathrm{x} 100 \mu \mathrm{m}$ beams with a $5 \mu \mathrm{m}$ gap, did not survive the SOG process, which produced a yield of zero. The heat generated during
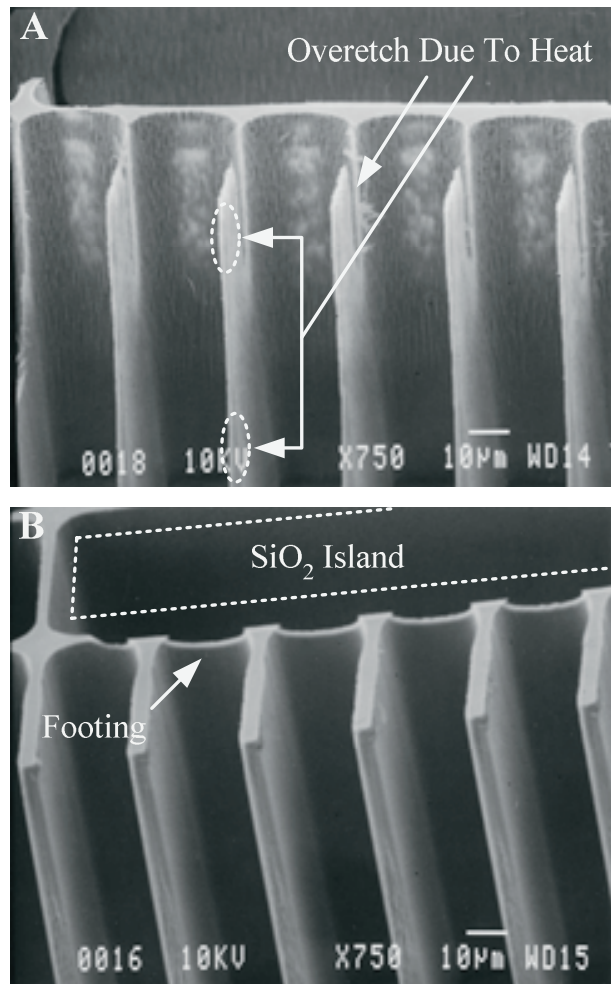

Figure 5. SEM of the bottom of comb fingers made using SOG (a) and SOS (b) The device made using SOG is severely overetched, while the SOS device shows a nice etch profile with mild footing.

DRIE causes the thin long beams of the Pirani gauge to be completely undercut and etched away.

Adhesive tape was used to break off the released resonators to expose the bottom for inspection. A SEM close-up of the bottom of the comb fingers is shown in Figure 5a SOG and Figure 5b SOS. The comb-fingers made using SOS show very mild signs of footing due to a nearby oxide island. However their etch profile turned out as expected, while the SOG comb fingers have been severely consumed by the uncontrollable etch due to overheating.

\section{ACKNOWLEDGEMENTS}

The authors thank Dr. Sangwoo Lee for his assistance. This project is partially supported by the Engineering Research Centers Program of the National Science Foundation under Award Number EEC-9986866.

\section{REFERENCES}

[1] J. Chae, H. Kulah, and K. Najafi, "A hybrid Silicon-On-Glass (SOG) lateral micro-accelerometer with CMOS readout circuitry," MEMS'02, Las Vegas, NV, (2002).

[2] N. Belov and N. Khe, "Using deep RIE for micromachining SOI wafers," $52^{\text {nd }}$ Electr. Comp. \& Tech. Conference, San Diego, CA, (2002).

[3] A. Sharma, F. M. Zaman, B. V. Amini, and F. Ayazi, "A high-Q in-plane SOI tuning fork gyroscope," IEEE Sensors, p. 467-70,Vienna, Austria, (2004).

[4] K. Ishihara, Y. Chi-Fan, A. A. Ayon, and M. A. Schmidt, "Inertial sensor technology using DRIE and wafer bonding with connecting capability," JMEMS vol. 8, pp. 403, (1999).

[5] Q. Y. Tong and U. Gosele, "Semiconductor Wafer Bonding," John Wiley \& Sons Inc, (1999). 